\title{
Importance of Tuberculosis (TB) Infection Prevention among Nurses and Patients at St. Dominic Mission Hospital
}

\author{
Article by Elizabeth Njovu \\ Nursing, Texila American University \\ E-mail: njovulisa@yahoo.com
}

\begin{abstract}
Tuberculosis infection control is the topic of great importance especially in developing countries where poor immunity, overcrowding, and some infections are a predisposing factor for Tuberculosis infection. The most common predisposing factors and measures to reduce the spread of TB infection from one person to the other are elaborated in this study. The findings reveal that even nurses taking care of the TB patients are at great risk of contracting the infection, hence strong TB infection prevention guidelines need to be strictly followed at all level of care.

Main Objective: To explore the knowledge and understanding of the qualified Nurses working in the out-patient department and medical ward in relation to Tuberculosis infection control and its impact on the hospital stay and patients outcome.

The method used: A cross-sectional study was conducted to identify predisposing factors to TB Infection. Data was collected by self-structured questionnaire of both closed and open-ended questions. A purposeful sample of 30 nurses from an out-patient department and male medical ward participated on a voluntary basis. I personally worked in these two departments to get the experience of what and how TB infection control is observed by nurses.

Results: 30 nurses participated: 19 females and 11 males; aged 21 to 50 years. Majority (82\%) diploma Holders, did registered nursing. Findings revealed that (75\%) appreciates the use of protective clothing - Masks while attending to TB infected patients while $25 \%$ did not, $45 \%$ finds it important to isolate those who are suspected to have TB and those having active TB, 100\% observed the guideline of keeping the doors and windows open for free movement of air.
\end{abstract}

Keywords: TB infection prevention, Environment control, Protective clothing, Isolation of the infected.

\section{Introduction}

Tuberculosis (TB) is an infectious disease that is caused by Mycobacterium tuberculin (MTB). Tuberculosis commonly affects the lungs, but can also affect other organs in the body. Most people especially those that have been exposed to the infection do not present with symptoms, this stage is known as a latent phase of tuberculosis. About $10 \%$ of this latent phase would develop into active disease. The classic symptoms of active TB are a chronic productive cough, most time with blood-stained sputum, fever, sweats, chest pain, anorexia and weight loss.

Tuberculosis is an airborne disease that is spread from one person to another when a person, with symptoms of active lung Tuberculosis cough, spit, speak, or sneeze. According to research, active TB infection is more common in $70 \%$ of people with HIV/AIDS and in those who have compromised immunity. Diagnosis of active TB is based on sputum examination (Smear microscopy, Gene-X-pert, and Culture), as well as microscopic examination and culture of body fluids. About $25 \%$ of people may not have any symptoms i.e. they remain "asymptomatic". (TB guidelines 2005)

According to the Zambia Ministry of Health, National TB and leprosy control program manual 2009 and the national Health Care workers TB evaluation protocol 2011, the national TB case notification rate was $337 / 100,000$ of the population. 
DOI: $10.21522 /$ TIJNR.2015.03.02.Art019

ISSN: $2520-3126$

\section{Purpose of the study}

The purpose of this study is to assess the requirements for the effective TB infection prevention and to identify an effective infection prevention policy and plan for St. Dominic Mission Hospital in Ndola. The project is based on the findings in the Hospital risk assessment and is consistent with the national Tuberculosis infection prevention guidelines for the patients and clients 2013.

It is also crucial for Nurses working in the outpatient department and medical ward because of their considerable involvement in the care of the TB patients. The issues to be explored in the study surround the understanding and knowledge of tuberculosis infection prevention and how Nurses can contribute to the effective TB infection prevention and patient's outcome.

\section{Background of the study}

Tuberculosis infection prevention depends on a number of factors. Other studies reveal that the most important risk factor globally is HIV; which account for 70\%. This is a significance problem in subSaharan Africa. (WHO Estimated HIV Prevalence in new TB cases, 2010) Only a few clients/patients without HIV will develop active TB disease. When a patient has both TB and HIV, priority is given to TB treatment before initiation of antiretroviral treatment.

Tuberculosis is associated with overcrowding, malnutrition, chronic lung disease and Smoking of cigarettes. Some illnesses like diabetes mellitus and habits like alcoholism can also increase the risk of developing tuberculosis.

In Zambia Tuberculosis prevention and control efforts rely primarily on the vaccination of infants, early detection of those infected and appropriate treatments of active cases. In children, Bacillus Calmette-Guérin vaccination has reduced the risk of getting the infection by $20 \%$ and the risk of latent phase turning into disease by $60 \%$. It is a national policy to screen all HIV-infected patients and all the exposed people for Tuberculosis, early detection and treatment have reduced the fatality of the diseases. The Ministry of Health through the help of PEPFAR has intensified the implementation of WHO 3I's which is:

- Intensified Case Finding

- Isoniazid Preventive Therapy

- Infection Control

\section{Practical aspect of tuberculosis infection prevention}

According to the research findings, the following were identified as practical ways to enhance TB infection prevention

- Personal hygiene: cover the mouth when coughing or sneezing, daily change of linen and clothing promotes good well being

- Good nutrition: eating a well-balanced diet will boost one's immunity that will help fight the infections

- Well ventilated and clean environment that prevent stagnation of air and bacterial growth. Open windows and doors will promote cross ventilation in the room. Prevent overcrowded.

- Isolation of the infected especially the $1^{\text {st }}$ two weeks of diagnosis and treatment when the bacteria are more active.

- Triage all those patients who are coughing, identify the coughers in the outpatient department give them face masks and attend to them as soon as possible.

- Practice hand wash before and after attending to every patient

- Decontamination and processing of linen and used instruments

- Proper disposal of waste using proper color codes

- Wearing of protective clothing

- Health education on personal hygiene to patients and caregivers 
Good planning, adequate funding and constant program monitoring and evaluation are very important in the TB infection prevention. Failure to adequately fund the program would risk the staff and patients being infected. Therefore the Hospital management needs to be committed to constantly and regularly provide resources through efficient budgeting and prioritizing.

\section{Research method}

The research was done at St. Dominic's Mission Hospital, Ndola district, Copperbelt province, Zambia. The research used a cross-sectional quantitative design, rationale being that cross-sectional is suitable as it saves time and resources (Saunders et al., 2007). Collected data gave details on TB infection prevention and control. Purposive sampling, a type of non-probability sampling, which is extremely useful was used to gain useful and rich, also in-depth information about nurses working in the outpatient department and medical wards, where nurses come in direct contact with TB patients.

Data collection method used was self-administered structured questionnaire of both open and closed ended questions. Questionnaires were hand delivered to 30 nurses and then hand-collected, to ensure confidentiality; and data collected were verified for quality. Apart from the questionnaire I personally worked in the outpatient department for one month and medical ward for another month. The information gathered is both from Nurses' experience and my own interaction with nurses and patients in the two departments

\section{Literature review}

Tuberculosis infection control program is based on the three levels of prevention, that is:

1. Administrative controls: This reduces the risk of exposure and is the number one and most important level of a TB infection control program. Administrative controls have the following activities:

- Assigning someone the responsibility and authority to coordinate TB infection control program at the health-care setting;

- Conducting a TB infection control risk assessment of the staff

- Developing and instituting a written TB infection-control plan to ensure prompt detection and treatment,

- Isolation and treatment of persons who are suspected or confirmed with TB disease;

- Ensuring the availability of recommended laboratory processing, testing, and reporting of results;

- Implementing effective work practices for managing patients who may have TB disease;

- Ensuring proper cleaning, sterilization, or disinfection of equipment that might be contaminated.

- Educating, training, and counseling HCWs, patients, and visitors about TB infection and disease;

- Annual screening of all health care workers

- Testing and evaluating workers who are at risk for exposure to TB disease;

2. Environmental controls: which prevents the spread and reduces the concentration of droplet nuclei. Adhering to the environmental factors for TB infection prevention would help a lot to reduce the spread of TB infection. The following factors reduce the spread of TB infection:

- Monitoring the flow of mechanical or natural air flow in the waiting area and examination room.

- Using posters and signs to remind patients and staff of proper cough etiquette (covering mouth when coughing) and respiratory hygiene.

- Signage to keep doors and windows open for free flow of air

- Avoid overcrowding of patients.

3. Personal Protective equipment: which further reduce the risk of exposure. Personal protective equipment when used together with other strategies of infection control reduces the risk of TB transmission to staff and others within the hospital. Masks and tissues are made available to the patients that are coughing.

Every patient suspected to have TB infection is nursed in an institution set up like a Hospital, Chest center or Chest clinic. In order to control TB infection among the patients who come to the hospital the following concepts should be considered seriously: 
DOI: $10.21522 /$ TIJNR.2015.03.02.Art019

ISSN: $2520-3126$

1. Good and well-structured organization: In the hospital, patients need to know who screens TB patients, who do the counseling, who dispenses the drugs

2. Proper planning and adequate staffing: Tuberculosis infection prevention activities require good planning for and adequate staff allocation for effective outcome

3. Systemic nursing and Health assessment of the patient: the nurse attending to patients with TB should have learned skills to systematic assess the patient for proper diagnosis and plan of patient care.

4. Proper History taking and physical examination, a detailed history and physical examination is obtained for the patient which aid in diagnosing the TB infection

5. Diagnosis and treatment: when the patient has been diagnosed with TB infection treatment are commenced immediately.

6. Prevention of complications of other systems: continuous monitoring of the patient with TB is very important to avoid other organs and body system getting affected. The patient is nursed in the hospital especially the $1^{\text {st }} 2$ weeks of treatment and in an isolation room

7. Proper documentation and reporting: the information is properly documented and kept well for future reference, or and to be used for reporting on program and patient outcome

\section{Recent study}

In the combined study by Dagmar Sissolak, Frederick Marais and Shaheen Mehtarhe on TB infection prevention and control's experiences of South African nurses, the authors describe that TB infection in South Africa is one that is characterized by the higher degree of TB and HIV co-infection. In their research, the authors assessed the nurses' experiences of factors influencing TB infection prevention and control practices to identify risks associated with potential ways of transmission. In their finding, they discovered that the nurses' had major concerns about the possible risk of TB transmission to both patients and staff. The authors' also discovered the following as factors that lead to non-adherence to infection prevention guidelines among nurses:

- Lack of facilities to isolate the TB-infected patient

- Inadequate personal protective equipment,

- Lack of a TB-IPC policy,

- Inadequate TB training for staff,

- Communication barriers between staff and patients,

- The work overload for nurses.

\section{Levels of TB infection prevention}

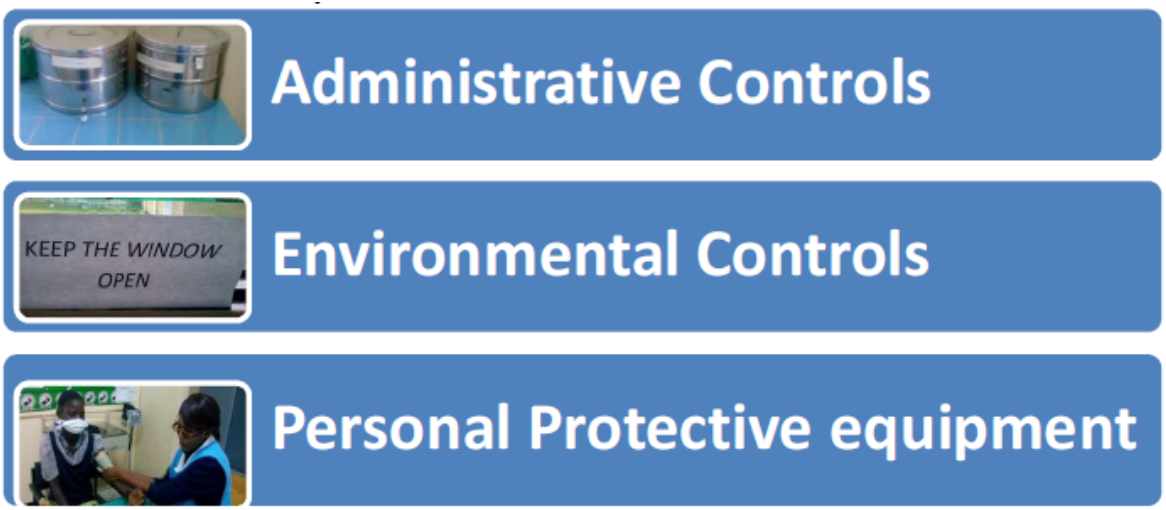




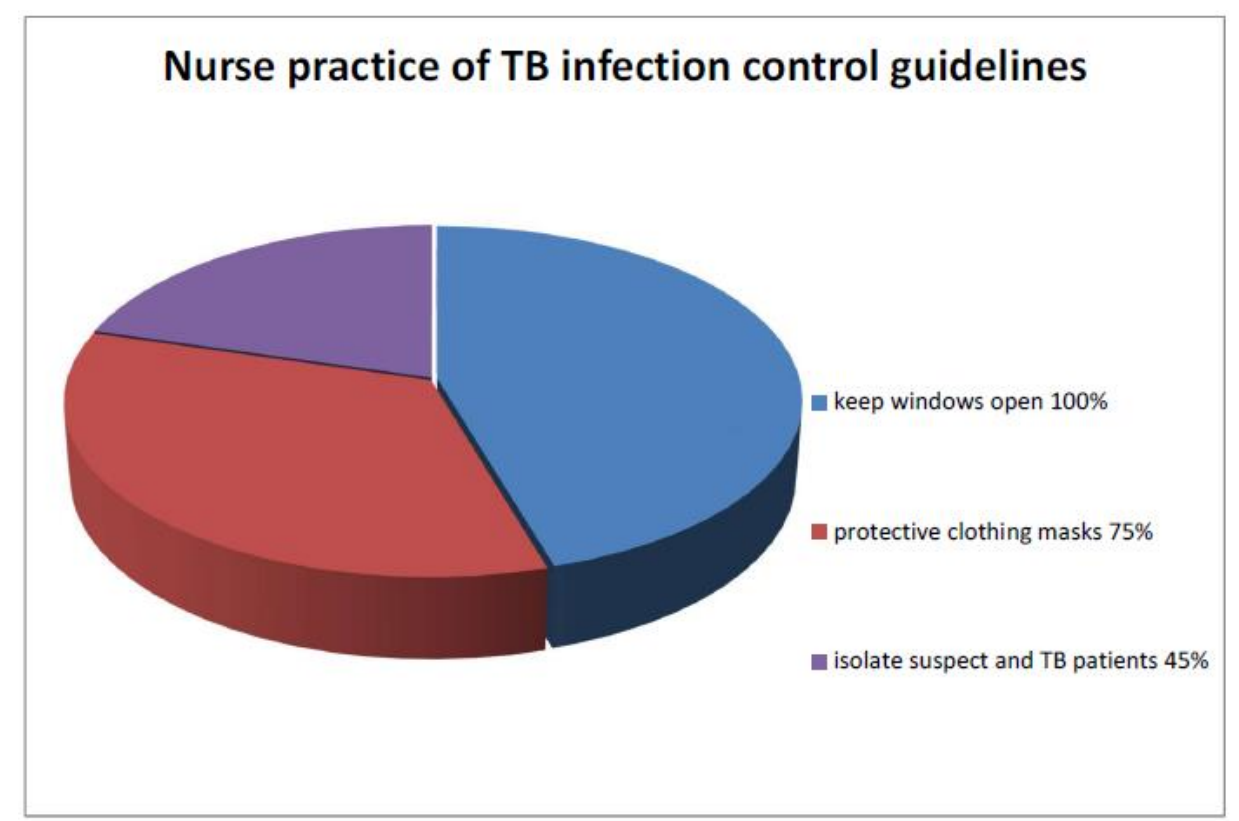

\section{Discussion}

Total numbers of study participants were 30 nurses: 19 females and 11 males; aged 21 to 50 years. Majority (82\%) diploma Holders, did registered nursing.

\section{Knowledge of nurses towards infection prevention and control}

Participants' had adequate knowledge about TB infection prevention as their responses gave this impression, and guidelines were available. The use of protective clothing was appreciated though they admitted that they were erratically supplied. The participants expressed that management did not priorities TB infection prevention in the budget.

The low response on isolating TB suspected patient signified noncompliance to TB infection prevention guidelines. Literature has specified that Tuberculosis infection control program is based on the three levels that are Administrative controls, Environmental controls, and Personal Protective equipment.

Equally, a study by Dagmar Sissolak, Frederick Marais and Shaheen Mehtahe on the TB infection prevention and control's experiences of nurses in South Africa, reveals that if guidelines are not followed, TB infection can be transmitted to both patients and staff. The authors' also discovered the main factors for nurses' non-adherence to infection prevention guidelines that could lead to the greater risk of transmitting infection from one person to the other:

\section{Recommendations}

Recommendations were based on findings from the research and analyzed data.

To healthcare providers (Nurses) at St. Dominic's Mission Hospital: Nurses are commended for the adequate knowledge on TB Infection Prevention. However, the following are some recommendations and suggestions that need to be implemented by all nurses:

- Should comply with TB infection prevention guidelines.

- Should continue working on improving attitude towards infection prevention, despite the pressure of work.

To St. Dominic's Mission Hospital management: The hospital is commended for having TB Infection Prevention guidelines and policy which are being used in various departments. The following are recommendations, so as to help nurses and other healthcare providers improve on TB Infection Prevention:

- Management should make TB infection prevention program a priority in the hospital budget. 
DOI: $10.21522 /$ TIJNR.2015.03.02.Art019

ISSN: $2520-3126$

- Should continue assigning a nurse to triage patients in the outpatients

To the Ministry of Health: The Ministry of Health is congratulated for continuous efforts being made on measures to improve health services to the society and recognize more and more the needs of healthcare providers.

However, the ministry should support and fund more research on TB Infection Prevention and deploy more nurses to the reduce workload on patients.

\section{Conclusion}

In order to reduce the risk of TB transmission to both the nurse and the clients at St. Dominic's Mission Hospital, an emphasis has been made to all nurses to strictly follow the guidelines and policies put in place by the hospital and those of the national. Despite the few nurses that have the negative attitude on following the guidelines, most of them appreciate and follow the guidelines. They are aware that not only patients are at risk of getting the infection but, they too are at even greater risk. One thing that the hospital administrator has done is the allocation of a nurse in the outpatient department to identify all patients with a cough, these patients are provided with a mask or a tissue to use while coughing. These patients are seen first in order to reduce the time spent with other patients in the outpatient department.

\section{References}

[1].Ajay K. Sethi, Charles W. Acher, Bruce Kirenga, Scott Mead, Curtis J. Donskey, Achilles Katamba M, 2012. Infection Control Knowledge, Attitudes, and Practices among Healthcare Workers, Kampala, Uganda.

[2].Brown, B., Crawford, P., Nerlich, B. \& Koteyko, N 2008. The habitus of hygiene: Discourses of cleanliness and infection control in nursing work, Social Science \& Medicine, Vol. 67, pp. 1047-1055.

[3].Claasens MM, van Schalkywk C, du Tolt E, Roest E, Lombard CJ, et al. Tuberculosis in health care workers and infection control measures at primary health care facilities in South Africa. PLoS One. 2013;8:e76272. doi: 10.1371/journal.pone.0076272.

[4].Dorothy Osigwe Chanda. Infection prevention manual for community and health care institutions in Developing countries.

[5].Infection control committee 2011. St. Dominic Mission Hospital TB infection control plan and policy.

[6].Katowa P. Muka to, C.M. Ngoma, M. Maimbolwa, 2007. Compliance with Infection Prevention Guidelines by Health Care Workers at Ronald Ross General Hospital, Mufulira district.

[7].Khaled M. Abd Elaziz and Iman M. Bakr, 2008, Cairo. Assessment of knowledge, Attitude.

[8].Kozier, Barbara F., et al., 2004. Fundamentals of Nursing: Concepts, Process, and Practice, 7th ed., Pearson Education South Asia, Pte Ltd.

[9].WHO policy on TB infection control in health-care facilities, congregate settings and households. Geneva: World Health Organization; 2009.

[10].Zambia Ministry of Health. National TB and leprosy control program manual 2009. 